Classification

Physics Abstracts

$07.75-42.30-82.80$

\title{
Microscope imaging by time-of-flight secondary ion mass spectrometry
}

\author{
Bruno W. Schueler \\ Charles Evans \& Associates, 301 Chesapeake Drive, Redwood City, CA 94063, U.S.A. \\ (Received March 20, 1992; accepted June 11, 1992)
}

\begin{abstract}
The concept of Secondary Ion Microscopy, introduced by Castaing et al. [1], is applied to a direct imaging time-of-flight (TOF) mass spectrometer. The ion optical configuration for a particular [2] stigmatic imaging TOF mass spectrometer is reviewed. A number of fundamental factors influencing the mass resolution in any TOF spectrometer are discussed. The question of where microscope imaging offers advantages over microprobe imaging in TOF Secondary Ion Mass Spectrometry is addressed.
\end{abstract}

\section{Introduction.}

Time-of-flight Secondary Ion Mass Spectrometry (TOF-SIMS) is rapidly developing into a powerful tool for surface microanalytical applications. Due to the capability of simultaneously detecting ions over a virtually unlimited mass range, high mass resolution, mass determination accuracy and ion transmission the technique permits applications which are not accessible with conventional dynamic SIMS. In particular, the ability to analyze a sample surface for inorganic and organic contamiants simultaneously with micrometer and sub-micrometer lateral resolutions provides new analytical dimensions.

The basic concept of time-of-flight SIMS is very simple. A pulsed primary ion beam bombards the sample surface, causing the emission of atomic and molecular secondary ions. The secondary ions are then electrostatically accelerated into a field free drift region with a nominal kinetic energy of:

$$
E_{\text {kin }}=e V_{0}=m v^{2} / 2,
$$

where $V_{0}$ is the accelerating potential, $m$ the mass of the ion, and $e$ its charge. Since lighter ions will have higher velocities than the heavier ones, they will arrive at the detector at the end of the drift region earlier than higher masses. The mass separation in such an instrument is thus obtained in the flight time $t$ from the sample to the detector which are roughly given by

$$
t=L_{0} / v=L_{0}\left(m / 2 e V_{0}\right)^{1 / 2}
$$

where $L_{0}$ is the effective length of the spectrometer. Since different ion types arrive sequentially at the detector, the operating conditions of such an instrument can be chosen such that (virtually) all 
secondary ions of a given polarity are detected and recorded in the mass spectrum. This efficient collection and detection of secondary ions by TOF-SIMS instruments minimizes the ion beam induced damage of the sample and results in a very sensitive technique for organic and inorganic surface analysis. Typical sample exposures in this static SIMS analysis mode are $\leq 10^{12}$ primary ions $/ \mathrm{cm}^{2}$ for mass analysis and $\leq 10^{13}$ primary ions $/ \mathrm{cm}^{2}$ for imaging.

Neighboring masses $m_{0}$ and $m_{0}+\Delta m=m_{0}(1+\gamma)$ can only be resolved as such if their time width and separation $\Delta t$ is sufficient. For small mass differences it is trivial to show from equation (2) that the relationship between flight times and mass resolution is:

$$
m_{0} / \Delta m=\gamma^{-1}=t_{0} / 2 \Delta t .
$$

It is obvious from equation (3) that in order to obtain high mass resolution a short primary ion pulse of duration, $t_{\mathrm{p}}$, has to be used since the smallest measurable $\Delta t$ is $t_{\mathrm{p}}$. The mass measurement in a TOF-SIMS instrument is achieved by (accurately) converting the flight time into exact mass by determining the flight time differences of the ions through the spectrometer.

Although conceptually very simple, there are a number of physical processes which require a more sophisticated design for a TOF spectrometer than merely having a long flight tube and fast detection electronics at the end. The two most extenuating phenomena are:

1. Secondary ions can be generated with non-zero initial kinetic energy and will thus acquire total kinetic energies which are larger than imposed by the accelerating potential. For sputtered atomic secondary ions, the initial kinetic energy distribution is centered around 5-10 eV with a tail of the distribution extending out to greater than $100 \mathrm{eV}$. Molecular ions are ejected with lower mean kinetic energy and are distributed over a much smaller energy range. The narrower energy distribution is a result of the kinetics of the sputtering process: collisions which impart high kinetic energy to the molecule will also result in high internal energy, thereby resulting in molecular fragmentation. Only those molecules which have sustained "gentle" collisions will survive and be detected. Inorganic molecular ions are thus observed to have initial kinetic energies in the 1-5 eV range with distributions rapidly falling off towards higher energies. A rule of thumb is that the more complex the detected molecule, the lower is its initial kinetic energy. Larger organic molecular ions, as the extreme, are typically emitted with initial energy distributions in the subeV region.

2. Secondary ions are emitted from the sample surface with an angular distribution with respect to the normal to the sample surface. Ions extracted by the accelerating field may enter the spectrometer with lateral momenta, i.e. kinetic energy, which causes them to diverge from the desired flight path through the mass analyzer.

A TOF mass spectrometer must therefore not only separate these ions according to their mass to charge ratio but also compensate for the initial kinetic energy and angular distributions caused by the sputtering and ionization process. The impact of these effects shall be discussed in more detail later in this paper.

TOF spectrometers provide the high ion transmission and collection efficiency which is crucial for analysis of the topmost monolayers of a sample. The rate of material removal on the other hand is very low. The time interval between pulses of the primary ion gun is determined via equation (2) by the mass range to be analyzed, the accelerating potential, and the length of the spectrometer. Typical repetition rates of the ion gun are approximately $20 \mathrm{kHz}$ for a mass range $0-400$ dalton, $e V_{0}=4.5 \mathrm{kV}$ and $L \approx 2 \mathrm{~m}$. With primary ion pulses containing of the order $10^{2}$ primary ions/pulse, the sputter rates are roughly $10^{7}$ atoms/s, i.e. not particularly well suited for depth profiling analysis such as in Dynamic SIMS. Some limited depth profiling capabilities can, however, be achieved by alternatingly analyzing a surface (with a pulsed beam), then sputter material with a continuous beam (no analysis), analyze all elements of this freshly exposed surface with a pulsed beam, etc. This method can provide rather useful qualitative information on 
impurity distributions e.g. in native oxide layers. The procedure is usefully restricted to rather shallow depth profiles (some 100 Angstrom) and sacrifices sensitivity for sputter rate improvement, however, with the advantage of detecting all secondary ion types during analysis of a given layer.

The lateral distribution of organic or inorganic contaminants is an important component in many surface analytical applications. The requirements for lateral image resolution vary from submicrometer to tens of micrometer, depending on the analytical task. Sub- $\mu \mathrm{m}$ imaging is clearly a domain most suited for microprobe instrumentation implementing a pulsed Liquid Metal Ion Gun (LMIG). Imaging in the region of $1-5 \mu \mathrm{m}$ on the other hand covers a large range of practical materials problems. This image resolution region has been extremely successfully covered by dynamic SIMS ion microscopes since their introduction by Castaing et al. [1]. An adaptation of the concept of stigmatic secondary ion imaging for organic and inorganic surface analysis of the topmost monolayers utilizing a TOF-SIMS ion microscope $[2,4]$ shall be discussed in this paper.

\section{A TOF-SIMS ion microscope system: experimental.}

A variety of very elegant mass analyzer configurations capable of providing isochronous (for angle, energy, and source extension) as well as achromatic spatial focusing of charged particles [5-9] has been proposed. Common to all these geometries is the combination of (field free) drift regions and electrostatic analyzers (ESAs). The aspect of stigmatic imaging in these instruments is presently only used in the sense that the (typical) 1:1 magnification image transport can provide high ion transmission. A stigmatic imaging mass spectrometer does, on the other hand, only qualify as an ion microscope if a magnified image of the emission profile from the sample surface is transported to the detector, i.e. necessitating the use of magnifying transfer optics. A schematic diagram of such a TOF secondary ion microscope is shown in figure 1.

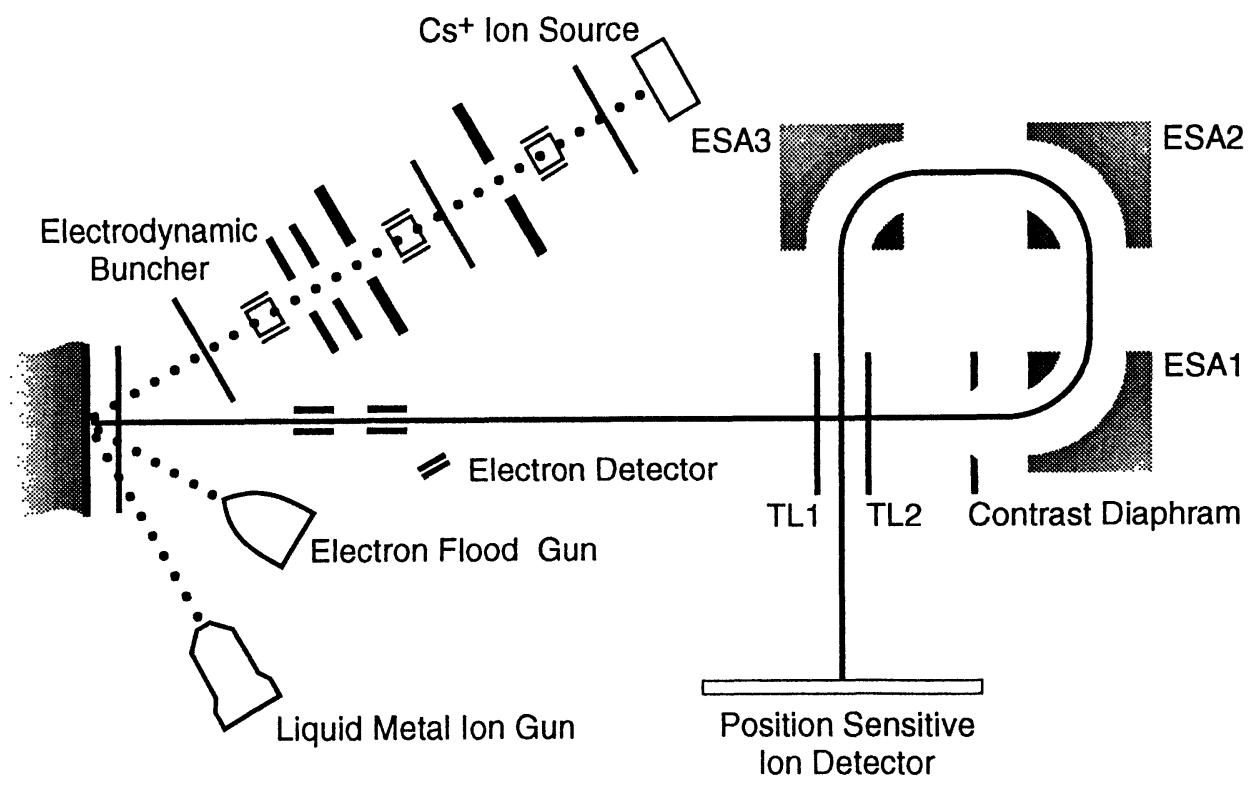

Fig. 1. - Schematic diagram of a stigmatic imaging TOF-SIMS mass spectrometer configured for microscope and microprobe imaging operation. 
A pulsed primary ion beam strikes the sample surface causing the emission of secondary ions. Secondary ions of the selected polarity are electrostatically extracted and focused by an immersion lens. The immersion lens activated in conjunction with one of two transfer lenses (TL1 and TL2) determines the total image magnification. The accessible image fields are approx. $70 \mu \mathrm{m}$ and $300 \mu \mathrm{m}$, magnified to $2.5 \mathrm{~cm}$ on the imaging detector, when transfer lens 2 or 1 are activated, respectively. Image resolutions of $1 \mu \mathrm{m}$ can be obtained. A stigmatic imaging system of three $90^{\circ}$ (quasi) hemispherical ESAs forms part of the ion optics and provides first order energy focusing for imaging and flight times with small residual second order aberrations. The final magnified secondary ion image is projected onto an imaging detector. The ESAs are fitted with field termination plates ("Herzog shunts") as well as "Matsuda plates" for electrostatic field shaping. A pulsing system controls a low energy electron gun and the extraction potential to provide charge neutralization of insulators with $15 \mathrm{eV}$ electrons during positive and negative secondary ion analysis [23].

Mass spectra are recorded by measuring the time difference between pulsing of the primary ion gun and the arrival of secondary ions on a fast dual microchannelplate (DMCP) detector at the end of the spectrometer by means of a multistop time-to-digital converter (TDC) or a single stop TDC of 156ps time resolution. A fast resistive anode encoder (RAE) is placed behing the DMCP detector to subsequently encode the $(x, y)$ arrival position of secondary ions on the detector. This position information may or may not be utilized in the analysis. The collection of image information is thus (correlated to but) decoupled from the time measurement. Ion imaging is achieved by either utilizing the $(x, y)$ image position of the secondary ions at the end of the spectrometer (microscope imaging) or by registering the $(x, y)$ raster position of the primary ion beam on the sample surface (microprobe imaging). The difference in microprobe and microscope imaging is merely in which type of position information is stored with the mass spectrum.

The pulsed $\mathrm{Ga}^{+}$LMIG is used for microprobe imaging where lateral image resolutions between 0.1 and $0.3 \mu \mathrm{m}$ are required. In this image acquisition mode the image position of the secondary ions as provided by the RAE is ignored and the raster position of the primary ion beam serves as image source.

In microscope imaging applications the image position of the secondary ions as provided by the RAE detector is utilized and the primary ion beam position ignored. Since the size of the probe beam is completely inconsequential in direct ion imaging, sub-ns ion pulses of high instantaneous current from either primary ion gun may be utilized. This allows for simultaneously achieving high mass resolution and $1 \mu \mathrm{m}$ lateral image resolution. The maximum data rate at which the $(x, y)$ information is collected is set by the RAE and is independent from the mass spectra acquisition. All position information is collected if the ion arrival times are separated by $200 \mathrm{~ns}$. If the ion arrival is spaced between 70 and $200 \mathrm{~ns}$, the arrival position of the first ion is encoded and the second one rejected. If the pulse spacing is between 25 and $70 \mathrm{~ns}$, both arrival positions are rejected. Only for ion arrivals within the $25 \mathrm{~ns}$ confusion time of the RAE system is an average arrival position is recorded.

The image and mass spectrum data acquisition system is configured to allow the storage of every detected set of position $(x, y)$ and time (mass) coordinates into a "raw data" file. From this raw data file it is retrospectively possible to:

a) recreate ion images for any arbitrary mass detected in the mass spectrum, and

b) recreate mass spectra from any arbitrary region in the ion images.

Up to 16 images are presently simultaneously recreated. Three sets of image regions (each consisting of up to 16 sub-regions) can be acquired in parallel into three different mass spectra. This procedure eliminates the necessity for preselection of ion images before the analysis and the complete analysis can be replayed at any time for further evaluation. It is additionally possible to monitor 16 preselected ion images and display mass spectra from three different regions of the image field while the analysis is in progress. 


\section{First order ion optics.}

Particle trajectories and flight times through mass spectrometers can be conveniently described by transfer matrices $[5,10,11]$ as motion relative to the ion optical axis. The coordinates $(x, \alpha)$ in the dispersion plane and $(y, \beta)$ give the perpendicular distances and inclination angles with respect to the spectrometer axis. If a reference particle has a mass to charge ratio $m_{0} / e$ and energy to charge ratio $E_{0} / e$, an ion of different mass and energy may be characterized as

$$
\begin{aligned}
& m / e=\left(m_{0} / e\right)(1+\gamma), \text { and } \\
& E / e=\left(E_{0} / e\right)(1+\delta) .
\end{aligned}
$$

Flight time differences can be described by the parameter $\tau$

$$
\tau=v_{0}\left(t-t_{0}\right)=L_{0}\left(t-t_{0}\right) / t_{0}
$$

where $t_{0}$ is the flight time of a particle traveling along the ion optical axis (a length $L_{0}$ ) with energy $e V_{0}$ ( $V_{0}$ being the accelerating voltage), mass $m_{0}$ and velocity $v_{0}$.

Following the description in reference [3] these matrices are in the first order approximation given as:

$$
\begin{aligned}
& {\left[\begin{array}{l}
x \\
\alpha \\
\gamma \\
\delta \\
\tau
\end{array}\right]=\left[\begin{array}{ccccc}
(x / x) & (x / \alpha) & (x / \gamma) & (x / \delta) & 0 \\
(\alpha / x) & (\alpha / \alpha) & (\alpha / \gamma) & (\alpha / \delta) & 0 \\
0 & 0 & 1 & 0 & 0 \\
0 & 0 & 0 & 1 & 0 \\
(\tau / x) & (\tau / \alpha) & (\tau / \gamma) & (\tau / \delta) & 1
\end{array}\right] \cdot\left[\begin{array}{c}
x_{0} \\
\alpha_{0} \\
\gamma \\
\delta \\
\tau_{0}
\end{array}\right]} \\
& {\left[\begin{array}{l}
y \\
\beta
\end{array}\right]=\left[\begin{array}{ll}
(y / y) & (y / \beta) \\
(\beta / y) & (\beta / \beta)
\end{array}\right] \cdot\left[\begin{array}{l}
y_{0} \\
\beta_{0}
\end{array}\right]}
\end{aligned}
$$

where $x_{0}, \alpha_{0}$, etc. and $x, \alpha$, etc. are the ion parameters before and after the ion optical element(s), respectively.

3.1 ION ENERGY FOCUSING AND FLIGHT TIMES. - TOF mass spectrometers always incorporate field free drift regions of some length $L_{0}$. Secondary ions produced by sputtering will always exhibit a spread $\Delta E_{0}$ in ion emission energy, resulting in particle energies $E=e V_{0}\left(1+\Delta E_{0} / e V_{0}\right)=$ $e V_{0}(1+\delta)$ after electrostatic acceleration to a nominal energy of $e V_{0}$. The energy spread will result in a time dispersion in the drift space of

$$
\begin{aligned}
& \left(t-t_{0}\right) / t_{0}=(1+\delta)^{-1 / 2}-1 \approx-\delta / 2 ; \delta<<1, \text { or since } \\
& (\tau / \delta) \delta=L_{0}\left(t-t_{0}\right) / t_{0}: \\
& (\tau / \delta)=-L_{0} / 2 .
\end{aligned}
$$

A particle having a kinetic energy a fraction $\delta=\Delta E_{0} /\left(e V_{0}\right)$ higher than the reference particle will arrive at a time equivalent to the flight time of an ion with energy $e V_{0}$ traveling only a distance $L_{0}-\left(L_{0} / 2\right) \delta=L_{0}+(\tau / \delta) \delta$. The first aim is then to introduce energy dispersive ion optical elements which apparently lengthen the effective flight path for higher energetic particles by the equivalent amount $(\tau / \delta)=+L_{0} / 2$ to compensate for this effect. 
Let us now consider the system of three electrostatic analyzers shown in figure 1. For sake of clarity it shall be assumed that the ESAs are hemispherical with radius $R$, a deflection angle of $90^{\circ}$ and fringe field corrections neglected. The transfer matrix for the single ESA is then [5]:

$$
[\mathrm{ESA}]=\left[\begin{array}{ccccc}
0 & R & 0 & R & 0 \\
-1 / R & 0 & 0 & 1 & 0 \\
0 & 0 & 1 & 0 & 0 \\
0 & 0 & 0 & 1 & 0 \\
2 & 2 R & R \pi & (3 \pi / 4-2) R & 1
\end{array}\right]
$$

The corresponding transfer matrix for a drift space of length $D$ is [5]:

$$
[D]=\left[\begin{array}{ccccc}
1 & D & 0 & 0 & 0 \\
0 & 1 & 0 & 0 & 0 \\
0 & 0 & 1 & 0 & 0 \\
0 & 0 & 0 & 1 & 0 \\
0 & 0 & D / 2 & -D / 2 & 1
\end{array}\right]
$$

The complete analyzer system may be represented as the product matrix

$$
[A]=[Z] \cdot[\mathrm{ESA}] \cdot[D] \cdot[\mathrm{ESA}] \cdot[D] \cdot[\mathrm{ESA}] \cdot[L]
$$

where $[L]$ and $[Z]$ are the matrices for drift spaces before the first and after the third ESA, respectively. The ESAs are assumed to be evenly spaced. Under these assumptions the non-zero coefficients of the complete transfer matrix for the energy compensation system in the deflection direction are:

$$
\begin{aligned}
& {[x / x]=D / R-Z D^{2} / R^{3}+Z / R} \\
& {[x / \alpha]=L D / R-R-Z L D^{2} / R^{3}+Z D / R+Z L / R} \\
& {[x / \delta]=R-D+Z D^{2} / R^{2}-Z} \\
& {[\alpha / x]=-D^{2} / R^{3}+1 / R} \\
& {[\alpha / \alpha]=L / R+D / R-L D^{2} / R^{3}} \\
& {[\alpha / \delta]=D^{2} / R^{2}-1} \\
& {[\gamma / \gamma]=1} \\
& {[\delta / \delta]=1} \\
& {[\tau / x]=2 D^{2} / R^{2}-2} \\
& {[\tau / \alpha]=2 R-2 D-2 L+2 L D^{2} / R^{2}} \\
& {[\tau / \gamma]=(Z+L) / 2+D+3 \pi R / 4} \\
& {[\tau / \delta]=2 R-2 D^{2} / R-D+9 \pi R / 4-(Z+L) / 2} \\
& {[\tau / \tau]=1}
\end{aligned}
$$

The system should, of course, be achromatic $([x / \delta]=0)$ as well as radially focusing $([x / \alpha]=0)$ and furthermore not introduce any image size dependent time dispersion $([\tau / x]=0)$. From the 
requirement $[\tau / x]=0$ it follows immediately that:

$D=R$,

From which achromatic focusing follows automatically.

Radial focusing is obtained for any combination

$Z+L=R=D$.

By arbitrarily choosing $Z=L=R / 2$, the transfer matrix in the deflection direction becomes:

$$
\left[\begin{array}{l}
x \\
\alpha \\
\gamma \\
\delta \\
\tau
\end{array}\right]=\left[\begin{array}{ccccc}
1 & 0 & 0 & 0 & 0 \\
0 & 1 & 0 & 0 & 0 \\
0 & 0 & 1 & 0 & 0 \\
0 & 0 & 0 & 1 & 0 \\
0 & 0 & R(3 / 2+3 \pi / 4) & R(9 \pi / 4-3 / 2) & 1
\end{array}\right] \cdot\left[\begin{array}{c}
x_{0} \\
\alpha_{0} \\
\gamma \\
\delta \\
\tau_{0}
\end{array}\right]
$$

The corresponding matrix in the perpendicular direction is:

$$
\left[\begin{array}{l}
y \\
\beta
\end{array}\right]=\left[\begin{array}{ll}
1 & 0 \\
0 & 1
\end{array}\right] \cdot\left[\begin{array}{l}
y_{0} \\
\beta_{0}
\end{array}\right] .
$$

This energy compensating system provides achromatic stigmatic focusing with unity image magnification. A schematic diagram of the achromatic ion transport through the analyzer system for two ions which travel along the spectrometer axis but having kinetic energies $e V_{0}$ and $e V_{0}(1+\delta)$ and the corresponding situation for ions starting inclined to the ion optical axis is shown in figure 2 .

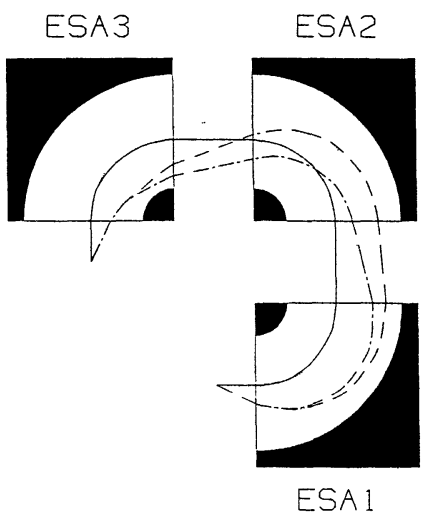

Fig. 2. - Schematic diagram of the stigmatic achromatic ion transfer through the energy analyzer system in the deflection direction. The ion transport is indicated starting from one point with $[\alpha, \delta=0],[a=0, \delta>0]$, $[\alpha<0, \delta=0]$, and $[\alpha<0, \delta>0]$.

The analyzer system provides the appropriate time dispersion with respect to the particle energy (faster particles have longer flight path than the slower ones) of:

$$
[\tau / d]_{\mathrm{A}}=R(9 \pi / 4-3 / 2) .
$$

In order to obtain complete energy compensation for the mass spectrometer system $[\tau / \delta]_{\text {sys }}=0$, additional beam transport optics having $[\tau / \delta]_{\text {trans }}=-[\tau / \delta]_{\mathrm{A}}$ may be introduced. 
3.2 EFFECTS OF THE ACCELERATING SECTION. - The preceding discussion did not include the influence of the accelerating section on the ion optical system. Image aberrations occurring in the accelerating space have been extensively reviewed by Slodzian $[12,13]$ and shall not be discussed here. Some aspects of time aberration due to the accelerating section have been discussed e.g. by Poschenrieder [5]. Since flight time aberrations introduced in the accelerating section have a major effect and will ultimately limit the performance section have a major effect and will ultimately limit the performance of any type of TOF spectrometer, a brief discussion seems appropriate.

Most TOF mass spectrometers incorporate an electrostatic accelerating section of length $z$. A particle which is sputtered with an initial energy $E_{0}$ at an angle $\alpha$ with respect to the surface normal has a flight time $t_{\text {acc }}$ through the accelerating space of:

$$
t_{\mathrm{acc}}=z\left(2 m / e V_{0}\right)^{1 / 2}\left\{\left(1+\delta \cos ^{2} \alpha\right)^{1 / 2}-\delta^{1 / 2} \cos \alpha\right\}
$$

where $\delta=E_{0} / e V_{0}$ and $V_{0}$ the acceleration potential. In a TOF spectrometer of length $L_{\mathrm{s}}$, the flight time error caused by the accelerating section alone for ions emitted with

$$
\alpha=0, \delta>0
$$

with respect to ions having no initial kinetic energy is:

$$
\begin{aligned}
& \Delta t_{\mathrm{acc}} / t_{0}=2 z\left\{(1+\delta)^{1 / 2}-\delta^{1 / 2}-1\right\} /\left\{2 z+L_{\mathrm{s}}\right\}, \text { and hence } \\
& {[\tau / \delta]_{\mathrm{acc}}=2 z\left\{(1+\delta)^{1 / 2}-\delta^{1 / 2}-1\right\} / \delta \approx z-2 z / \delta^{1 / 2}}
\end{aligned}
$$

The first term in $[\tau / \delta]_{\text {acc }}$ is trivial to correct for by shortening the spectrometer by $2 z$. A severe flight time error is, however, introduced due to $2 z / \delta^{1 / 2}$, which is not entirely correctable by flight path adaptation.

The solid line in figure 3 shows the dependence of $\Delta t_{\mathrm{acc}} / t_{0}=[\tau / \delta]_{\mathrm{acc}} \delta / L_{\mathrm{s}}$ as a function of the relative ion energy spread $\delta$, for an accelerating section of $z=4 \mathrm{~mm}$ and an effective spectrometer length $L_{\mathrm{s}}=2 \mathrm{~m}$. For reference, a $\Delta t_{\mathrm{acc}} / t_{0}=5 \times 10^{-5}$ corresponds to a mass resolution of 10000 . If no corrections for the $[\tau / \delta]_{\text {acc }}$ were provided, the TOF spectrometer could only provide high mass resolution for organic molecules, for which the initial energy spread $\Delta E_{0}$ is known to be primarily in the sub-eV region [22]. Severe deterioration of the mass resolution would be encountered for atomic secondary ions, having initial axial energy distributions peaking at several $\mathrm{eV}$ and extending into the several $10 \mathrm{eV}$ region. The mass resolution could only be improved by operating at very high extraction fields which often imposes high voltage discharge problems at the sample surface or by rigorous energy filtering via energy slits in ESA systems or limitation of the energy band pass in ion mirror [14-16] systems.

It is clear that at least for a limited bandwidth of initial ion kinetic energies the dependence of $[\tau / \delta]_{\text {acc }}$ on $\delta$ can be linearly approximated in a rather straightforward manner by an appropriate adaptation of drift paths and/or the effective length of the energy compensating system. One criterion for such a linear approximation may be based on the assumption that an energy range from $2 \mathrm{eV}<E<20 \mathrm{eV}$ is the most critical region to be compensated for in atomic ion analysis. This approximation requires for the above given accelerating section a spectrometer compensation $[\tau / \delta]_{\text {spec }} \approx 8.5 \mathrm{~cm}$ which is indicated by the straight line in figure 3 . This approximation would result in a mass resolution limit of approx. 15000 up to $\delta<6 \times 10^{-3}\left(\Delta E_{0}<30 \mathrm{eV}\right.$ for $\left.V_{0}=4500 \mathrm{~V}\right)$. This represents a respectable performance limit for practical purposes.

The energy compensating system discussed in section 3.1 consisting of drift regions and 3 ESAs was for sake of clarity based on ideal hemispherical analyzers without fringe fields. The system 


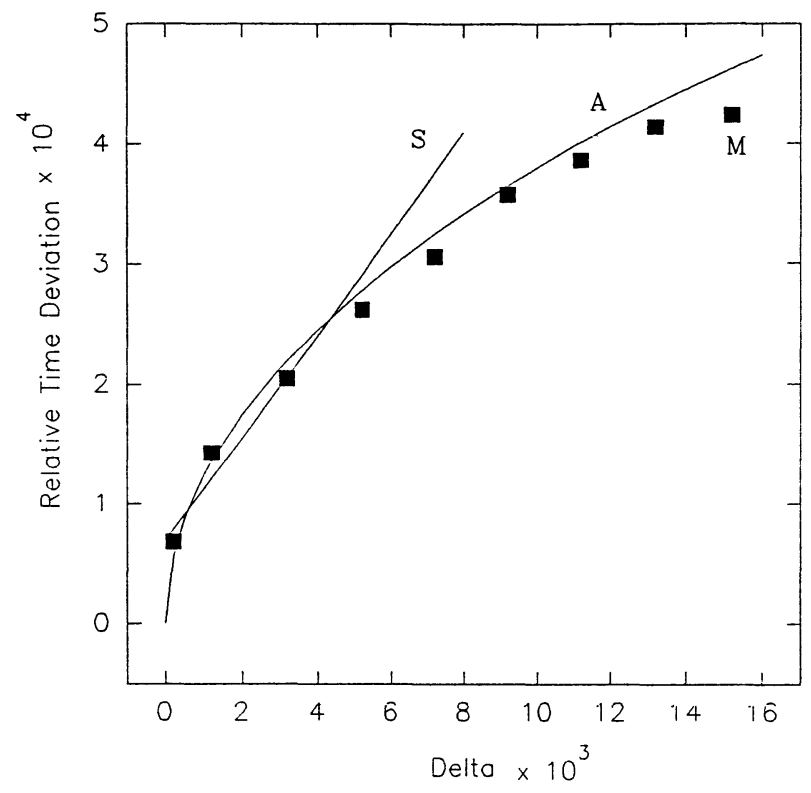

Fig. 3. - Time dispersion $\Delta T_{\mathrm{acc}} / t_{0}$ caused by the initial axial ion energy spread in an accelerating section $z=4 \mathrm{~mm}$ for a TOF spectrometer of $L=2 \mathrm{~m}$. The solid curve (A) indicates the calculated effect, the solid straight line $(S)$ indicates a linear approximation of the calculated curve with $[\tau / \delta] \approx 8.5$. The filled squares (M) indicate experimentally obtained data points.

used in practice is equipped with field terminators on the entrance and exit side of the electrostatic analyzers as well as "Matsuda plates" above and below the electrode gaps. This arrangement allows for a very fine adjustment of the field shape in the ESAs and a very close matching of the coefficient $[\tau / \delta]_{\text {spec }}$ in the spectrometer to the shape required by the accelerating space. The filled squares in figure 3 indicate the experimentally determined points of the energy compensation curve for the mass spectrometer section. This shape is in part obtained by operating the ESAs in a slightly toroidal as opposed to strictly spherical field shape. This rather close matching allows for flight time energy compensation over a range of $\delta \approx 14 \%$ or $\Delta E_{0} \approx 60 \mathrm{eV}$ in initial ion energy at $V_{0}=4500 \mathrm{~V}$ with mass resolution limit of better than 15000 . It should be mentioned that no noticeable influence on the imaging performance was observed under these spectrometer tuning conditions.

The time dispersion caused by the initial ion energy spread for particles emitted with $\alpha=0$ in the accelerating section can be well compensated for. The simplified case of ion emission angles of $\alpha=0^{\circ}$, however, not encountered in practice for sputtered atomic ions. For particles emitted with $E_{0}>0$ and $\alpha>0$ the flight time dispersion with respect to a particle emitted with the same $E_{0}>0$ but $\alpha=0$ caused in the acceleration section is

$$
\Delta t_{\mathrm{acc}} / t_{0}=2 z\left\{\left(1+\delta \cos ^{2} \alpha\right)^{1 / 2}-\delta^{1 / 2} \cos \alpha-(1+\delta)^{1 / 2}+\delta^{1 / 2}\right\} /\left(2 z+L_{\mathrm{s}}\right) .
$$

An energy compensating system is only sensitive to the total ion energy, whereas the accelerating section will exhibit a time dispersion with respect to the emission angle $\alpha$. The effect of the ion emission angle on the mass resolution limit $\left(t_{0} / 2 \Delta t_{\mathrm{acc}}\right)$ is illustrated in figure 4 for $\delta=1,2$, and $4.5 \times 10^{-3}$ for an accelerating gap of $z=4 \mathrm{~mm}$ and a total flight path $L_{\mathrm{s}}=2 \mathrm{~m}$. The exact magnitude of the angle dependent flight time error is difficult to estimate since it depends on the 
emission characteristics of the secondary ion under consideration. In the extreme, considering $\delta=$ $2 \times 10^{-3}\left(E_{0}=9 \mathrm{eV}\right.$ at $\left.V_{0}=4500 \mathrm{~V}\right)$, a TOF spectrometer operating at $100 \%$ ion transmission would have to be either $\approx 4$ times longer $(6 \mathrm{~m} !)$ or the acceleration section $\approx 4$ times shorter $(1 \mathrm{~mm}$ !) to safely assure mass resolutions better than $\approx 6000$ for atomic ions such as $\mathrm{Al}$ or $\mathrm{P}$ (including primary ion pulse width). None of the above is practical.

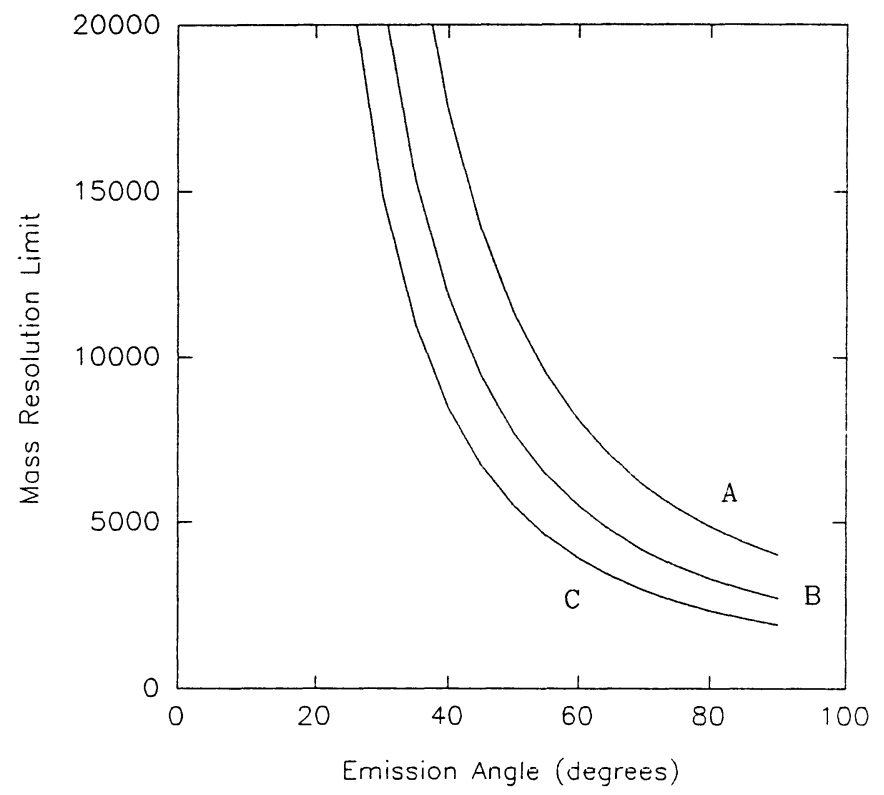

Fig. 4. - Dependence of the mass resolution limit as a function of the ion emission angle $\alpha$ imposed in an accelerating section of $z=4 \mathrm{~mm}$ and spectrometer length $L=2 \mathrm{~m}$. The three curves A, B and C represent ions with $\delta=1,2$, and $4.5 \times 10^{-3}$, respectively.

In reality, the angular acceptance of the spectrometer is limited by the ion extraction and beam transport optics and apertures. If the primary ion duration is short enough, mass resolution around 7500 may still be expected for atomic ions of the mass resolution limit set by the spectrometer is around 10000 . Under these conditions, assuming a $\cos \alpha$ lateral velocity distribution and that $\approx 65 \%$ of the secondary ions have $E_{0} \leq 10 \mathrm{eV}$, one can still expect a secondary ion transmission of $\approx 45 \%$ using $\alpha \approx 45^{\circ}$ (for $\delta \approx 0.002$ or $\Delta E_{0}=10 \mathrm{eV}$ at $V_{0}=4500 \mathrm{~V}$, see Fig. 4).

3.3 THE MICROSCOPE SYSTEM. - Microscope imaging implies that a magnified image of the secondary ion emission profile from the sample is transported to the detector. This is most conveniently accomplished by implementing an extraction lens and at least one transfer lens in conjunction with the energy compensating system. A schematic diagram of the magnified image transport from the sample, through the electrostatic analyzer section and to the detector is shown in figure 5.

The extraction optics consists of an accelerating section formed by the sample surface and the entrance aperture of an Einzel lens. The extraction lens projects a magnified image of the ion emission profile into the first field free region of the mass spectrometer. The image is transported via transfer lens into the first ESA, where close to its exit aperture an image is formed. Another 


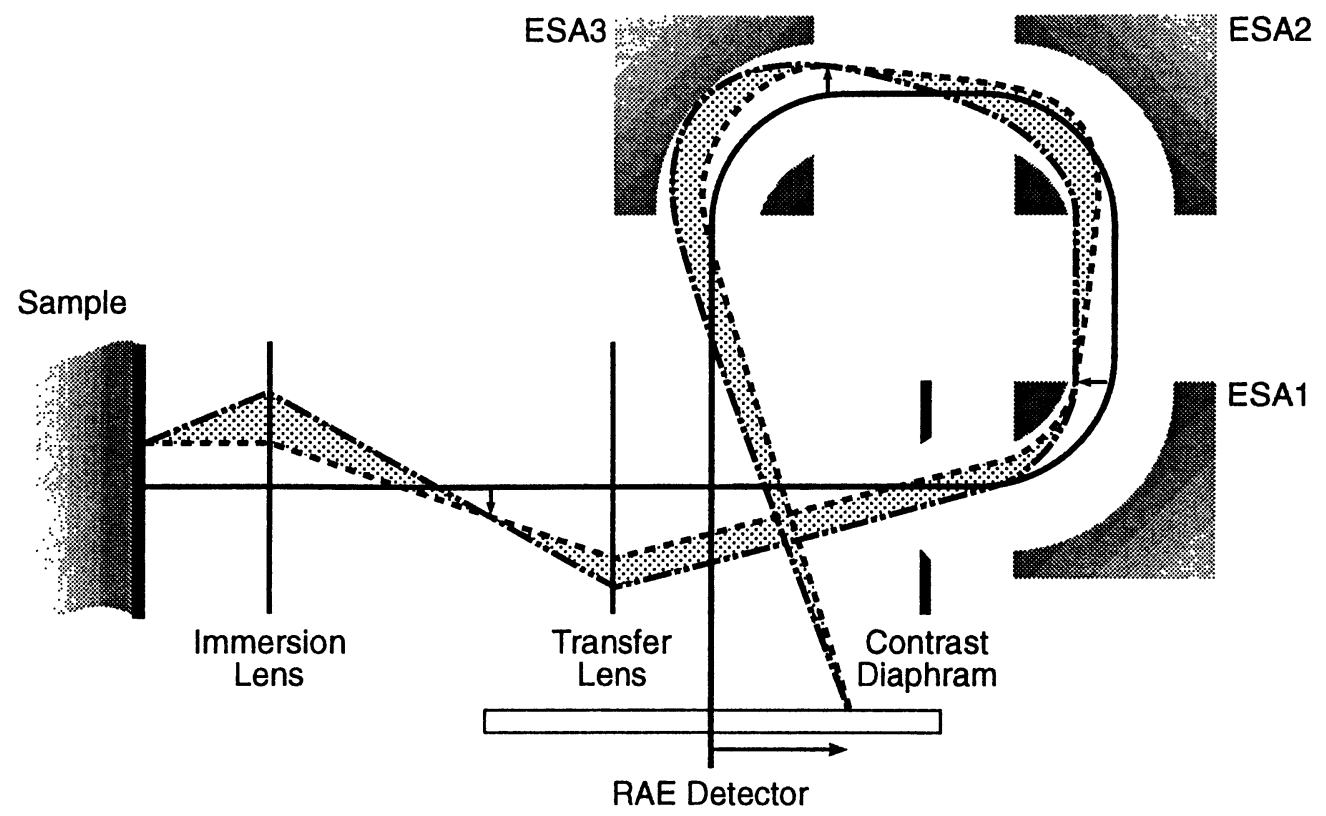

Fig. 5. - Schematic diagram of the magnified secondary ion image transport by means of immersion lens, transfer lens and energy analyzer system through the mass spectrometer. Ions starting from an off-axis point with different angles are indicated.

intermediate image is formed close to the entrance of the third ESA, which projects the final magnified ion image onto the position sensitive detector. An angle defining aperture can be placed into the crossover in front of the first ESA. All lenses are operated in the accelerating mode to minimize chromatic aberrations [17] of the image and flight time dispersion. The spectrometer system including extraction lens is calculated to accept secondary ions emitted parallel to the sample surface with $36 \mathrm{eV}$ initial kinetic energy at an accelerating potential of $V_{0}=4500 \mathrm{~V}$. This corresponds to a half angle acceptance of approx. $90 \mathrm{mrad}$ for the mass spectrometer after the accelerating section and ensures high secondary ion transmission (of the order $\geq 90 \%$ ) for some applications.

The ultimate image resolution is determined by the chromatic blur caused in the accelerating section $[12,13]$ of the mass spectrometer and chromatic and spherical aberrations of the ion optical system. The chromatic aberration fundamentally introduced in the accelerating section is significant for atomic secondary ion imaging and dominates over the image aberrations introduced in the mass spectrometer. This image aberration can only be counteracted by limiting the angular acceptance of the mass spectrometer and can be achieved by placing a "contrast diaphragm" into a secondary ion crossover (here: in front of ESA\#1).

On the other hand, for direct ion imaging applications of organic secondary ion species the effect of the accelerating section can be neglected since the ion energy distributions are typically in the sub-eV regime. In this situation the ultimate image resolution is, for practical purposes, primarily limited by the resolution of the imaging detector. High resolution microscope imaging of organic molecules is thus possible at close to $100 \%$ ion transmission.

Let us very briefly estimate the best expected lateral resolution in the direct ion imaging operation and the corresponding secondary ion transmission. According to Slodzian [10] the diameter 
of the aberration spot around the virtual sample image is given by:

$$
d_{\mathrm{a}} \approx\left(2 z \Delta E_{0} / V_{0}\right) \sin \alpha(1-\cos \alpha) .
$$

This aberration spot will ultimately limit the obtainable lateral resolution, aberrations of the ion optical system neglected. Let us assume that for typical sputtered atomic secondary ions approx. $70 \%$ of the axial energy distribution is contained in $0<E_{0}<15 \mathrm{eV}$ and that the lateral velocity distribution follows a $\cos \alpha$-relationship. For an accelerating gap of again $z=4 \mathrm{~mm}$, and $V_{0}=4500 \mathrm{~V}$ extraction voltage, the spot $d_{\mathrm{a}}$ in equation (15) reaches a value of approx. $4 \mu \mathrm{m}$ for an emission angle of $40^{\circ}$, given $\Delta E_{0}=15 \mathrm{eV}$. The expected transmission under the above assumptions is then of the order $0.7 \cdot \sin 40^{\circ} \approx 45 \%$. If lateral image resolutions of approx. $1 \mu \mathrm{m}$ are required under otherwise same conditions, the acceptance angle will have to be decreased to $\approx 25^{\circ}$, corresponding to $\approx 30 \%$ transmission. These imaging requirements are very compatible with the conditions necessary to obtain high mass resolution (for any TOF instrument with similar parameters) when anlyzing atomic secondary ions.

\section{Results.}

4.1 MASS RESOLUTION. - The practical constraints for obtaining high mass resolution in atomic secondary ions analysis with a TOF mass spectrometer have been discussed in section 3.2. With near to complete (axial) ion energy compensation the mass resolution will be primarily affected by the ion emission angle. The accepted lateral ion energy can be controlled by the contrast diaphragm and thus be adapted to the mass resolution required by the analysis.

Figure 6 shows a section around mass 56 of a positive secondary ion mass spectrum taken from a contaminated silicon wafer surface. Contamination levels for $\mathrm{Fe}$ on this wafer were independently determined by Total Reflection X-Ray Flourescence (TXRF) analysis to be in the $10^{11} \mathrm{~cm}^{2}$ range. The experimental conditions were such that lateral ion energies $\geq 6 \mathrm{eV}$ were rejected, a maximum energy band acceptance of $\approx 200 \mathrm{eV}$ (above accelerating potential), and a primary ion pulse width of $\approx 470 \mathrm{ps}$ fwhm as measured on $\mathrm{H}^{+}$secondary ions. The estimated secondary ion transmission under these conditions is $>45 \%$ for atomic ions. The mass resolution on Fe is approx. 8000 fwhm. As seen from the mass spectrum in figure $6,{ }^{56} \mathrm{Fe}^{+}$is easily separated from the ${ }^{28} \mathrm{Si}_{2}{ }^{+}$ molecular interference. Additionally, secondary ion signals due to $\mathrm{SiCO}, \mathrm{CNO}_{2}, \mathrm{SiCH}_{2}, \mathrm{C}_{3} \mathrm{OH}_{4}$, $\mathrm{C}_{3} \mathrm{NH}_{6}$, and $\mathrm{C}_{4} \mathrm{H}_{8}$ were identified with mass determination accuracies of better than $2 \mathrm{mamu}$. At full lateral ion energy acceptance (estimated transmission $>90 \%$ ), the mass resolution for atomic ions decreases to approx. 4000 which is not quite sufficient to separate $\mathrm{Fe}$ from $\mathrm{Si}_{2}$, whereas the mass resolution for organic ion species remains unaffected.

Figure 7 shows a section of a positive secondary ion mass spectrum taken from a film of Fomblin oil which was deposited on a Si wafer surface. The mass resolution obtained from the $\mathrm{C}_{2} \mathrm{~F}_{5}{ }^{+}$ secondary ion is seen to be approx. $15000 \mathrm{fwhm}$. The experimental conditions were the same as used for the mass spectrum shown in figure 6, except no lateral ion energy filtering was employed. As expected, the use of an emission angle limiting aperture (not shown) did not affect the mass resolution or the measured ion transmission. The main factors influencing the mass resolution or organic ion species are primary ion pulse width, ion detector timing errors and the roughness of the sample surface.

4.2 MiCROSCOPE IMAGE RESOLUTION. - Figure 8 shows three positive secondary ion images taken over a $300 \mu \mathrm{m}$ diameter region of an integrated circuit surface. Figure 8a shows the $\mathrm{Al}^{+}$ secondary ion image and the ${ }^{71} \mathrm{Ga}$ image in figure $8 \mathrm{~b}$ indicates the localization of $\mathrm{GaAs}$ structures 


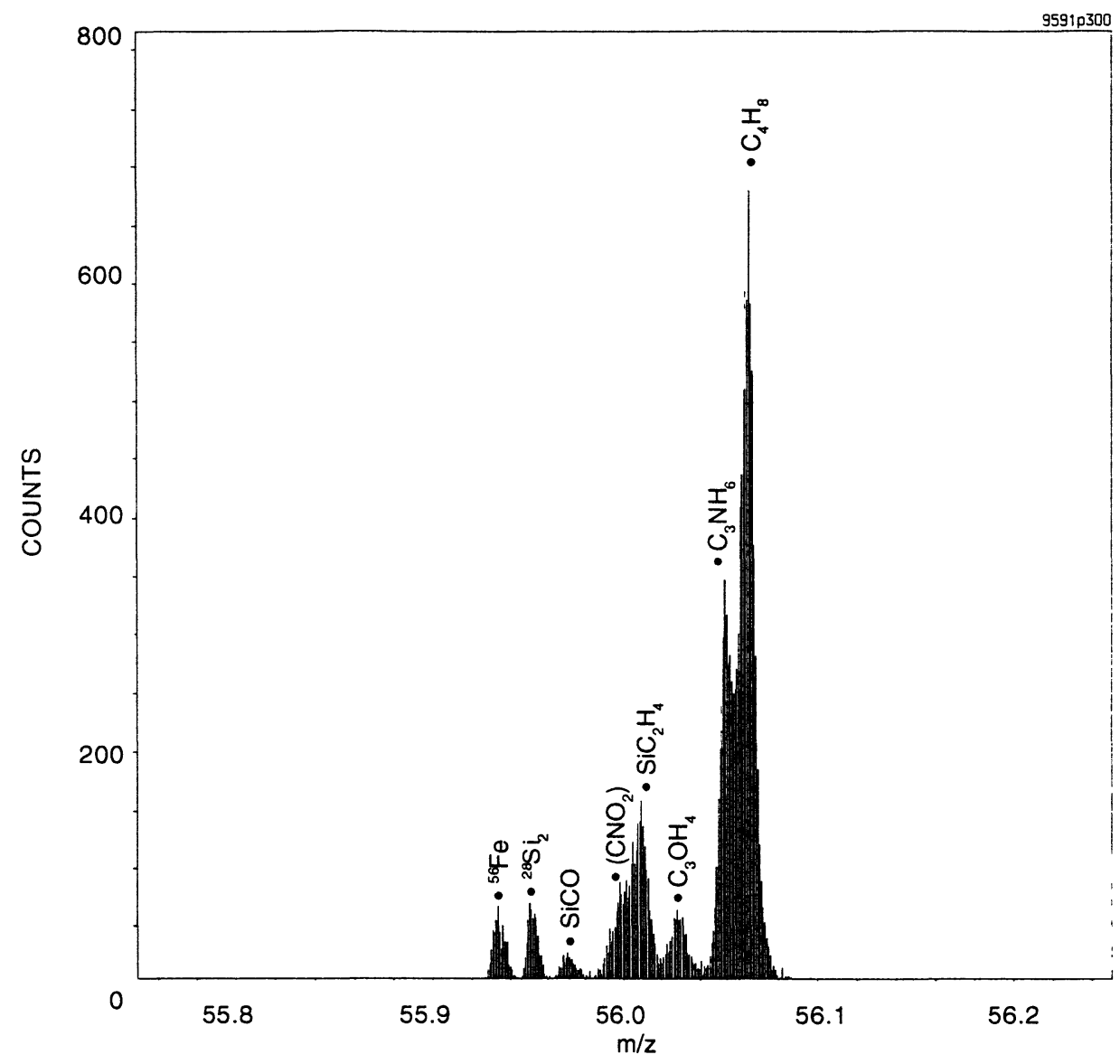

Fig. 6. - Section of a positive secondary ion mass spectrum around mass 56 obtained from a silicon wafer. The Fe contamination level was $\approx 10^{11} \mathrm{~cm}^{2}$, as determined by TXRF. The mass resolution at Fe is approx. 8000 fwhm.

on the device. An isolated localized contamination of $\mathrm{Cu}$ on the integrated circuit is indicated in figure 8c. All images were simultaneously recorded. The images were obtained at $4500 \mathrm{~V}$ accelerating potential and by activating transfer lens 1 (see Fig. 1) in conjunction with the immersion lens. The lateral resolution with the $300 \mu \mathrm{m}$ image field is limited to approx. $3 \mu \mathrm{m}$ due to the digitization of the imaging information from the position sensitive detector. All ions with energy components of $<6 \mathrm{eV}$ parallel to the sample surface were accepted. This image field is typically used for surface contamination analysis of semiconductors and other larger area analyses.

Higher microscope image resolutions are obtainable using a $70 \mu \mathrm{m}$ diameter image field by activating transfer lens 2 in conjunction with the immersion lens. The detector limited lateral resolution is then of the order of $1 \mu \mathrm{m}$. Figure 9 shows positive secondary ion images of $\mathrm{Al}$ (Fig. 9a) and ${ }^{71} \mathrm{Ga}$ (Fig. 9b) taken from a $70 \mu \mathrm{m}$ diameter area inside the image field of figure 8. The linescan through the ${ }^{71} \mathrm{Ga}$ ion image shows a lateral resolution of approximately $1 \mu \mathrm{m}$, as one pixel corresponds to approx. $0.5 \mu \mathrm{m}$. The poorer image definition to the right is due to the integrated circuit structure and not due to image aberrations. For this analysis, ion energies $<1.5 \mathrm{eV}$ parallel to the sample surface were accepted (approx. $20^{\circ}$ emission angle for $15 . \mathrm{eV}$ ions). The atomic ion 


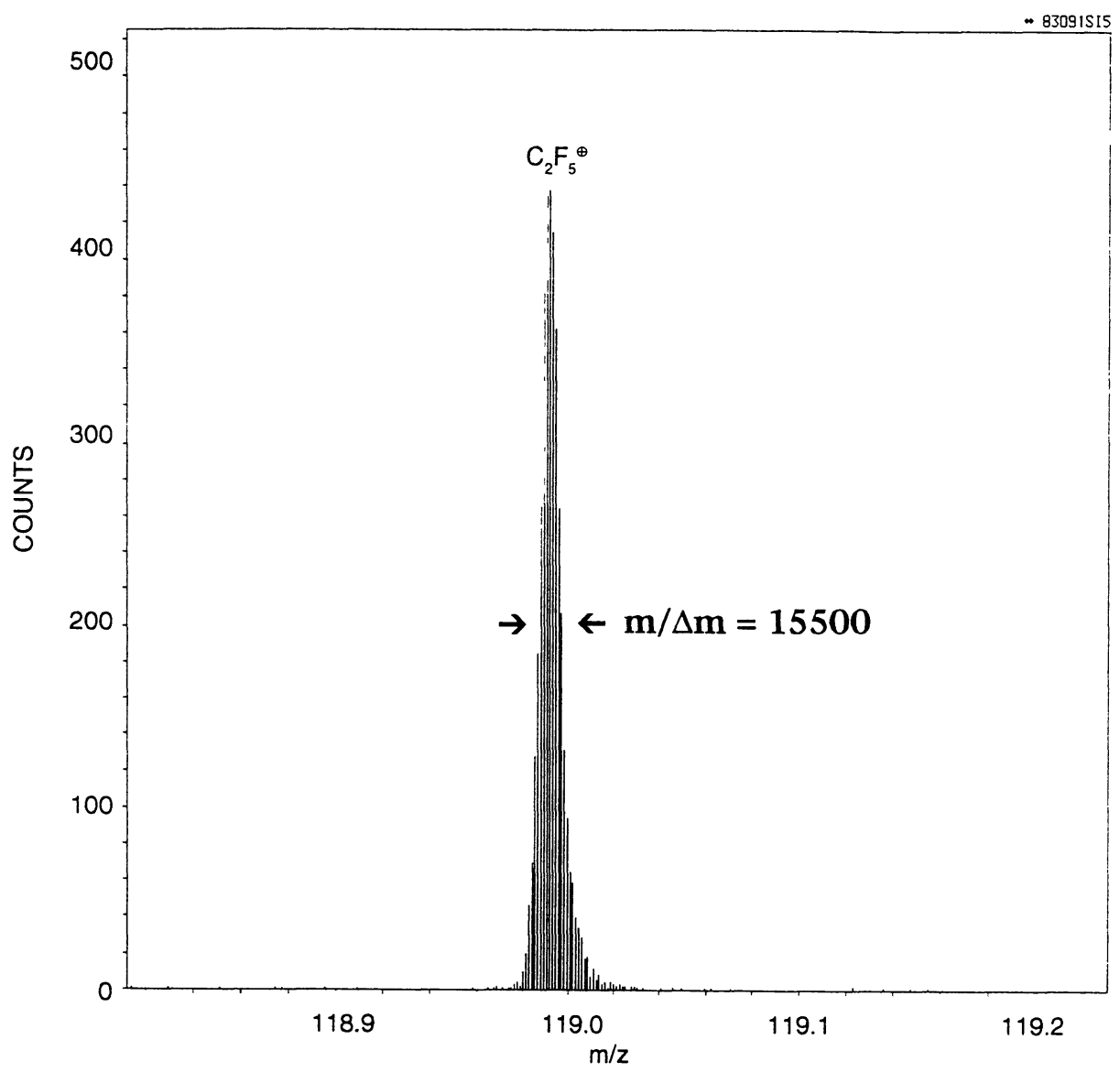

Fig. 7. - Example of $\approx 15000$ (fwhm) mass resolution for $\mathrm{C}_{2} \mathrm{~F}_{5}{ }^{+}$secondary ions. The sample was Fomblin oil dispersed on a silicon wafer.

transmission under these conditions is estimated to $\approx 20 \%$, whereas the organic ion transmission is not measurably affected.

An example of organic secondary ion imaging is given in figure 10. The sample was a mixture of epinephrine and norepinephrine crystals dispersed over an indium surface. A $300 \mu \mathrm{m}$ diameter image field was analyzed and no lateral ion energy filtering employed. Figures 10a and 10b show the lateral distribution of the protonated quasimolecular ions of epinephrine and norepinephrine, respectively. The differences in ion distribution in the two images clearly confirm the presence of the two sample constituents. This trivial example demonstrates the usefulness of (any) ion imaging as an additional diagnostic tool for mass spectra interpretation of mixtures. Differences in lateral ion distribution clearly indicate the presence of different components. Overlapping images either indicate fragments of the same molecule or lateral correlation of compounds. The availability of all ion images in TOF analyses can provide the tool for such data evaluation. 

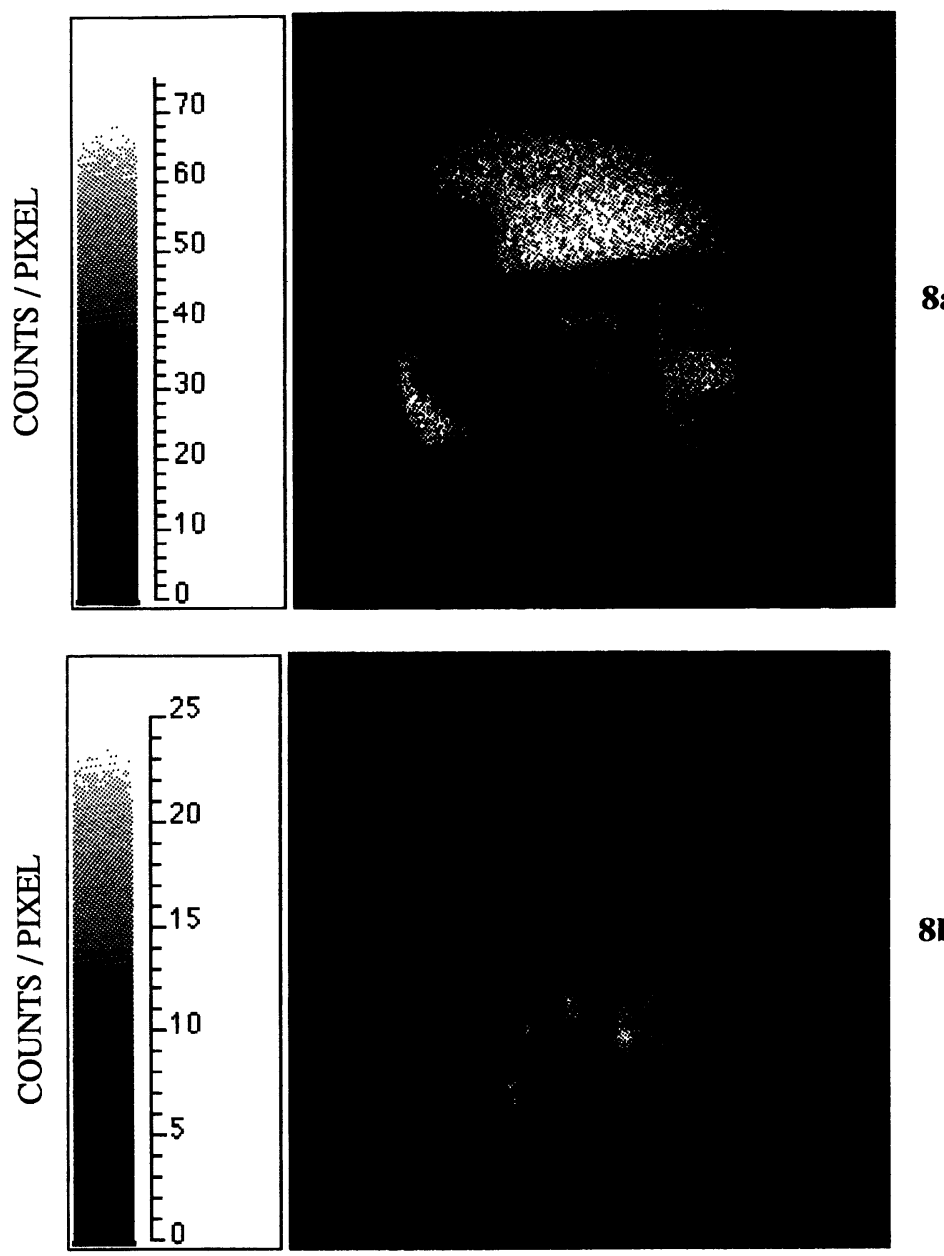

$\mathbf{8 b}$

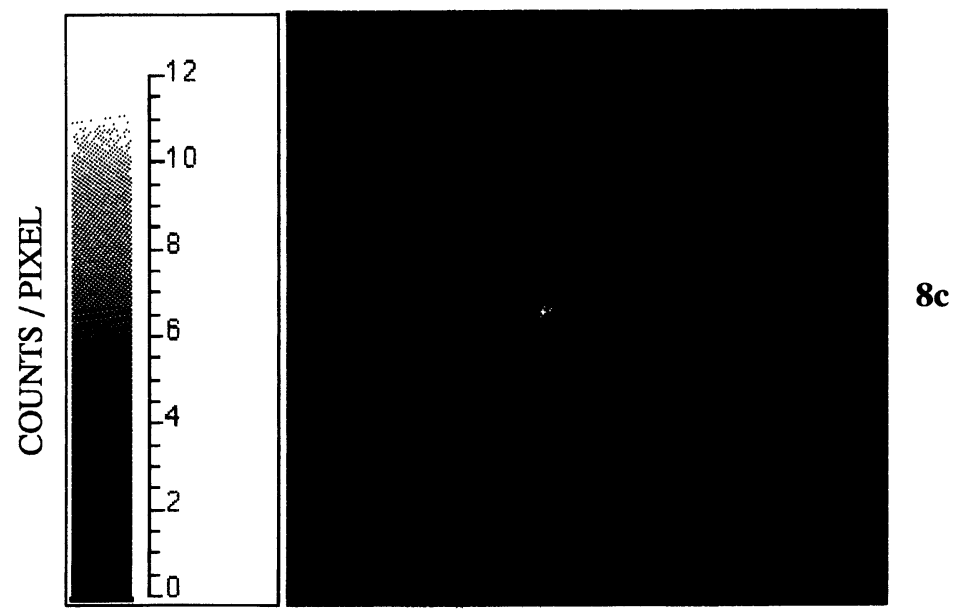

Fig. 8. - Positive secondary ion images of $\mathrm{Al}$ (Fig. 8a), Ga (Fig. 8b) an $\mathrm{Cu}$ (Fig. 8c) obtained from an integrated circuit surface. The analyzed sample area is $300 \mu \mathrm{m}$ in diameter. The intensity scale indicates the number of counts detected per pixel. 

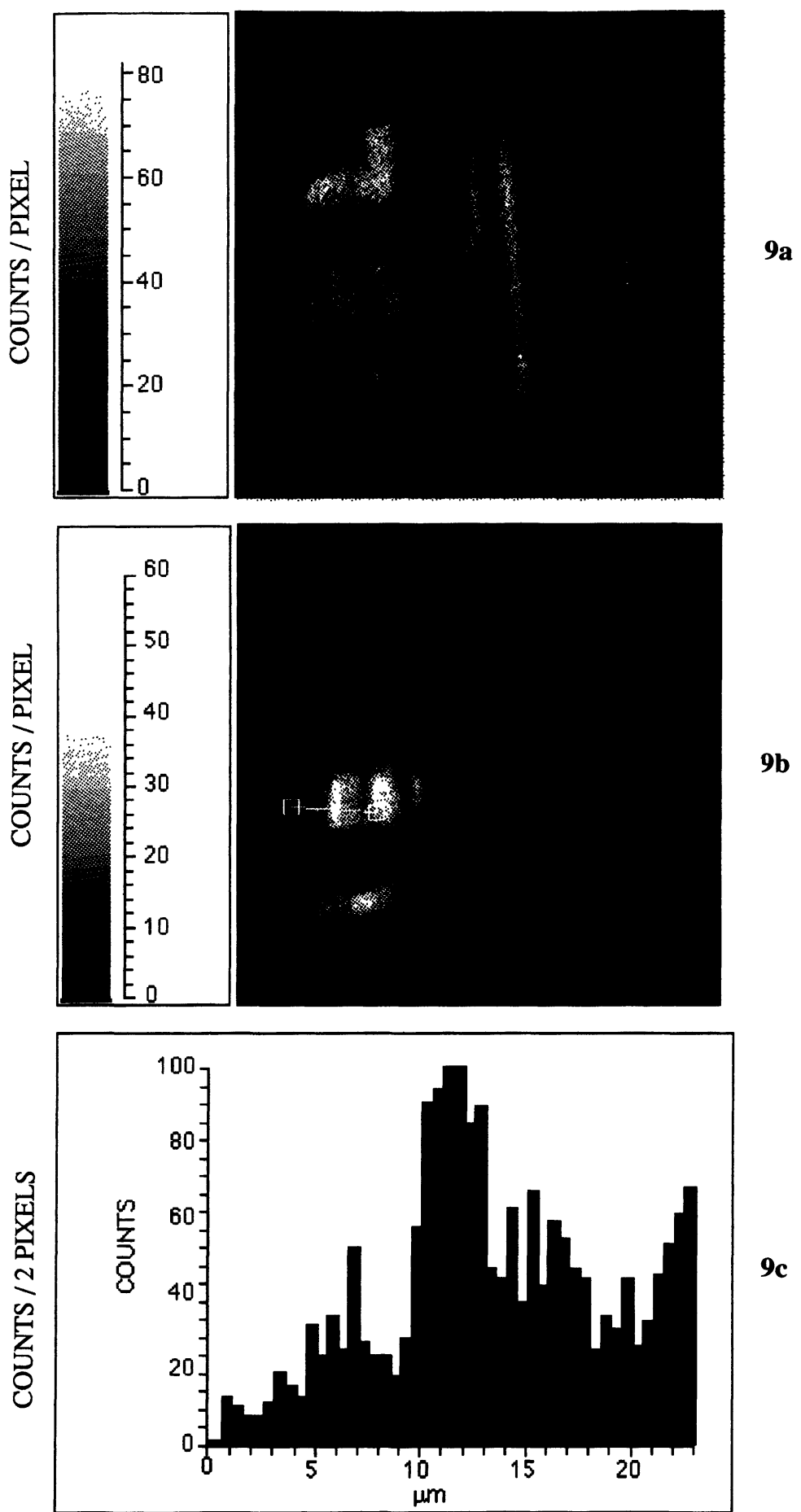

9c

Fig. 9. - Positive secondary ion images of $\mathrm{Al}$ (Fig. 9a) and Ga (Fig. 9b) obtained over a $70 \mu \mathrm{m}$ image field from an inegrated circuit. The linescan (Fig. 9 c) through the $\mathrm{Ga}$ ion image indicates a lateral image resolution of $1 \mu \mathrm{m}(1$ pixel $=0.5 \mu \mathrm{m})$. Linescan width is 2 pixels. 


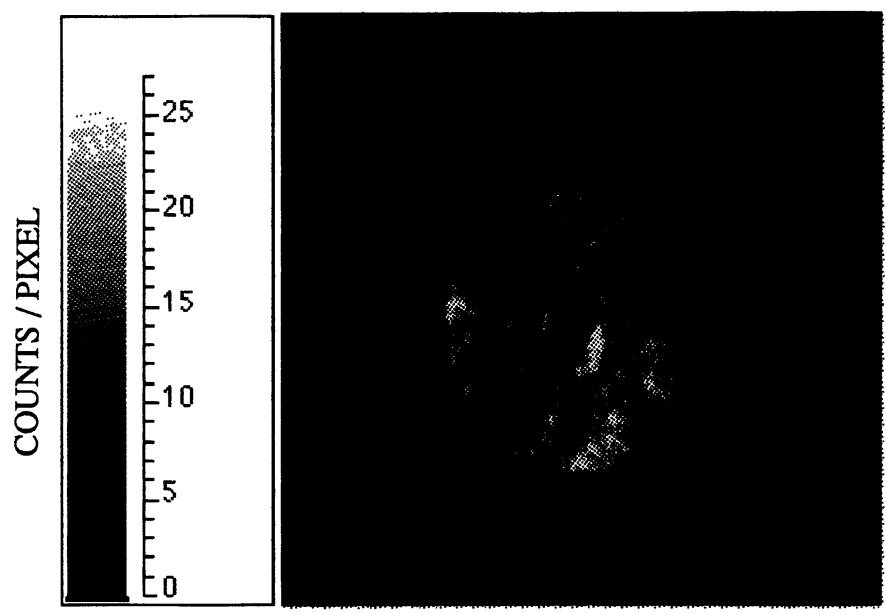

$10 \mathbf{a}$
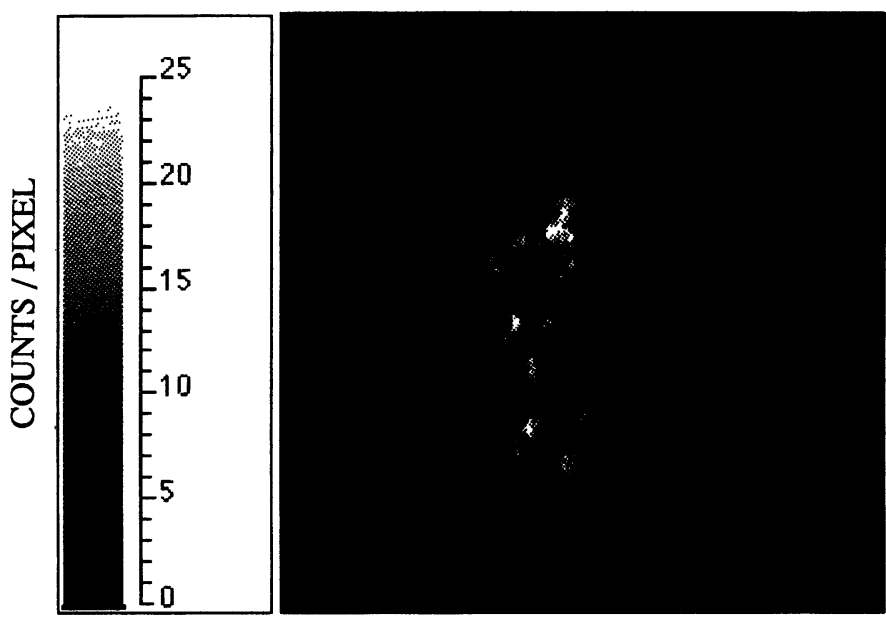

10b

Fig. 10. - Positive secondary ion images of the protonated quasimolecular ions of epinephrine (Fig. 10a) and norepinephrine (Fig. 10b) from a mixture of the two compounds dispersed on an indium substrate. The image field diameter is $300 \mu \mathrm{m}$.

\section{Why TOF-SIMS microscope imaging?}

The limitations in the imaging performance are either determined by the extracting and secondary ion optics or the diameter of the primary ion beam, depending whether the imaging information is obtained from the secondary ion image (microscope) or the raster position of the primary ion beam (microprobe), respectively. The analytical requirements alone can determine which type of image acquisition is most suitable for providing appropriate results.

In TOF-SIMS surface analysis, sub-micrometer image resolutions are most reasonably obtained using a pulsed Liquid Metal Ion Gun (LMIG). This imaging mode provides image resolutions of better than $0.2 \mu \mathrm{m}$. A typical Ga LMIG operating at $25 \mathrm{keV}$ beam energy provides $60 \mathrm{pA}$ continuous current into $\mathrm{a} 0.06 \mu \mathrm{m}$ spot and $500 \mathrm{pA}$ into $\mathrm{a} \approx 0.2 \mu \mathrm{m}$ spot. In the pulsed operation, necessary for TOF-SIMS analysis these currents translate into 0.375 and $3.125 \mathrm{ions} / \mathrm{ns}$, respectively. Pulsing of the LMIG typically deteriorates the spot size somewhat and practical lateral 
resolutions available with pulsed beams are of the order 0.1 and $0.3 \mu \mathrm{m}$ for low and high current operation, respectively. At this point it has to be considered that the best measurable mass resolution in a TOF instrument is given by the duration $t_{\mathrm{p}}$ of the primary ion pulse via $m / \Delta m \approx t / 2 t_{\mathrm{p}}$. Shortening of the primary ion pulse width decreases the number of primary ion contained in the pulse and results in an increase in analysis time. The achievable mass resolution is thus inversely proportional to the analysis time. Taking the above ion currents as typical examples, it is easy to estimate the mass resolution limit imposed by the necessary pulse width and the corresponding number of primary ion per pulse. Table I lists the number of primary ions available per pulse for a given required mass resolution assuming a mass spectrometer of $L=2 \mathrm{~m}$ and $V_{0}=3000 \mathrm{~V}$. Longer mass spectrometers could accept longer ion pulses but the highest repetition rate for the primary ion gun $\left(\approx\left(2 e V_{0} / m\right)^{1 / 2} / L_{0} ;\right.$ see Eq. (2)) decreases proportionally when the same mass range is to be analyzed, i.e. analysis times stay roughly the same.

Table I. - Number of primary ions per pulse available from a typical liquid metal ion gun for different requirements in mass resolution and image resolution.

\begin{tabular}{|c|c|c|c|c|}
\hline $\begin{array}{c}\text { mass } \\
\text { resolution } \\
\text { at mass }\end{array}$ & $\begin{array}{c}\text { lateral } \\
\text { resolution } \\
{[\mu \mathrm{m}]}\end{array}$ & $\begin{array}{c}\text { dc-current } \\
{[\mathrm{pA}]}\end{array}$ & $\begin{array}{c}\text { pulse } \\
\text { width } \\
{[\mathrm{ns}]}\end{array}$ & $\begin{array}{c}\text { primary } \\
\text { ions/pulse } \\
\text { (average) }\end{array}$ \\
\hline 500 at 500 & $\approx 0.1$ & 60 & 58 & 22 \\
1000 at 43 & $\approx 0.1$ & 60 & 7 & 2.5 \\
5000 at 56 & $\approx 0.1$ & 60 & 2 & 0.75 \\
500 at 500 & $\approx 0.3$ & 500 & 58 & 183 \\
1000 at 43 & $\approx 0.3$ & 500 & 7 & 22 \\
5000 at 56 & $\approx 0.3$ & 500 & 2 & 6 \\
\hline
\end{tabular}

In analysis situations where unit mass resolution up to mass 500 is sufficient, the available number of primary ions is entirely reasonable for analysis applications requiring the highest lateral resolution $(\approx 0.1 \mu \mathrm{m})$. This type of analysis is often used for determining lateral distributions of (mostly organic) contaminants at small scales or with extremely structured samples (such as fibers) where the large depth of focus provided by sub- $\mu$ m beams is of great advantage. The mass spectral analysis is via known fingerprint spectra of the compound in question. It has to be pointed out that under these operating conditions the surface image contrast will suffer somewhat, particularly for atomic ion imaging, since a separation of organic hydrocarbon contaminants from atomic ions is not entirely achieved. This analysis situation is rather similar to static SIMS quadrupole mass spectrometry, however with much higher sensitivity (all ions can be detected) and better mass resolution, but somewhat degraded lateral resolution.

The situation is somewhat different if higher mass resolution is required, e.g. in the vicinity of 12000 which is typically necessary to resolve the always present hydrocarbon ion signals from atomic ions or from oxygenated species. For lateral resolutions of $\approx 0.1 \mu \mathrm{m}$ only 2.5 primary ions/pulse are available which immediately shifts the required analysis times into rather prohibitive regions. If the analysis situation requires this lateral resolution, no alternative choice is available. If the lateral resolution can be in the $0.3 \mu \mathrm{m}$ region, acquisition times with a microfocused beam are still acceptable. 
In situations where high mass resolution $(>5000)$ is required, e.g. to separate $\mathrm{Fe}$ from $\mathrm{Si}_{2}$, the available number of primary ions is at best 7 ions/pulse. The use of an LMIG in those situations can then only be justified by the requirements for lateral resolution and the fact that the TOF-analysis is extremely surface sensitive. For any type of microprobe imaging it is trivially advantageous that the mass spectrometer has the highest possible ion transmission compatible with the required mass resolution (see Sect. 3.2).

If moderate lateral resolution of 1-2 $\mu \mathrm{m}$ are sufficient for the analysis, the direct microscope imaging operation becomes advantageous. The image resolution is completely independent of the size of the primary ion beam. It is therefore rather easily possible to produce sub-ns pulsed primary ion beams containing $\gg 80$ primary ions/pulse and achieve high mass resolution $(\geq 7500$ at mass 28) and good image resolution simultaneously within reasonable acquisition times. Microscope imaging can always be achieved without any loss in information at the maximum permissible count rate which is determined by the dead time of the imaging detector ( $\approx 200 \mathrm{~ns})$. Imaging by pulsed LMIGs in the high mass resolution regime at moderate image resolutions is limited by the same low instantaneous primary ion currents listed in table I. Even for true single ion counting the acquisition speed can be expected $>10$ times faster with the microscope mode than using a pulsed LMIG. The microscope imaging mode at high mass resolution and sensitivity is particularly advantageous in the analysis of organic surfaces where image aberrations are virtually nonexistent. This type of analysis capability is one of the great strengths of TOF-instruments compared to magnetic mass spectrometers. For very structured sample surfaces the image resolution the microscope imaging capability will be negatively affected due to differences in the microscopic fields on the sample surface. Imaging with focused primary ion beams (the complementary microprobe mode) will, in this particular instance, offer advantages in image performance. In this context it seems also appropriate to point out that the mass resolution of any TOF spectrometer will deteriorate due to potentially large fluctuations of the extraction fields around pronounced topographical structures of the sample surface.

For direct microscope imaging of atomic secondary ions at lateral resolutions better than $5 \mu$ $m$ the chromatic blur of the virtual sample image will necessitate appropriate lateral ion energy filtering via a contrast diaphragm. The spectrometer transmission is correspondingly decreased for atomic species (not for organic contaminants) but is still expected to be in the vicinity of $\approx 30 \%$ even for lateral resolutions around $1 \mu \mathrm{m}$. The moderate loss in transmission can be counterbalanced by an increase in the average primary ion current. Again, the limit in acquisition speed without loss in information is set by the imaging detector. If a loss in image information is acceptable, the instantaneous count rate can be increased, resulting in counting discrimination primarily in the most intense peaks (matrix ions).

In situations where image resolutions poorer than $\approx 5 \mu \mathrm{m}$ are sufficient, the advantage of TOF microscope over microprobe imaging disappears. High current focused ion guns capable of approx. $5 \mu \mathrm{m}$ lateral resolution are reported [16]. In either imaging mode an increase in data acquisition speed from (close to) single ion counting to multiple ion counting is necessary.

Hight mass resolution work by TOF mass spectrometry requires the use of time-to-digital converters (TDC) of some $100 \mathrm{ps}$ time resolution and the corresponding detection electronics typically consisting of preamplifiers and constant fraction discriminators. The best pulse pair resolution presently possible by such timing electronics is in the vicinity of $5 \mathrm{~ns}$. This implies that in analyses where many secondary ion are created per pulse, typically only the lightest ion at a given nominal mass is detected and multiple ions of the same type cannot be resolved and are counted as a single event. Distortions of the mass spectra are consequently occurring [19]. These distortions might be somewhat correctable via isotopic corrections (if available), but become extremely cumbersome at ion arrival rates of some 100 per pulse within one mass and recovery times of detectors become important. A compromise will have to be made between data acquisition speed 
and mass spectral distortion. Bearing in mind the above mentioned effects for time measurement, tradeoffs in imaging performance are apparent. Microscope imaging detectors presently have confusion times $\approx 25 \mathrm{~ns}$ (wrong position is recorded) and discrimination times of $70 \mathrm{~ns}$ where multiple counts occurring in this time period will be discarded and appear as no count in the image. For microprobe imaging the limitation is the $\approx 5 \mathrm{~ns}$ deadtime of the timing electronics and multiple counts in this time interval will be recorded (equally inaccurate) as a single event. With these distortions in mind, qualitative imaging in the microprobe image acquisition mode can still be possible at $>5$ times higher speed, provided the primary ion current is available.

\section{Conclusion.}

Stigmatic imaging secondary ion mass spectrometers offer the flexibility to select between microscope and microprobe imaging operation, depending on which image acquisition mode is the most suitable for a particular analytical problem. The concept of secondary ion microscopy in conjunction with a high performance mass analyzer offers clear advantages in imaging applications in the $\approx 1-5 \mu \mathrm{m}$ region requiring high mass resolution compared to microprobe imaging instruments, as implemented to date. For aplications requiring sub- $\mu$ m lateral resolution, microprobe technology is the imaging method of choice. Stigmatic secondary ion transport can offer an advantage due to the intrinsically high transmission of such instruments which can be adapted as required by the mass resolution necessary for the analysis. There is no particular advantage or disadvantage to direct ion imaging in applications requiring high mass resolution and pooer than $5 \mu \mathrm{m}$ lateral resolution compared to the microprobe imaging mode. The development of faster position sensitive detectors will further increase the data acquisition speed in direct ion imaging.

TOF mass spectrometry is at present the only technique providing the collection of all mass over a virtually unlimited mass range and all image information possible during the analysis. Microscope instrumentation can provide ion imaging with large or uncorrelated probe beams, e.g. imaging during matrix assisted laser desorption [20] (using a defocused laser spot) of large protein molecules. Another potential application is in imaging with $\mathrm{MeV}^{252} \mathrm{Cf}$ fission fragment induced desorption [21], where the useful ion yield for organic molecules is significantly higher than with keV-primary ion bombardment.

\section{Acknowledgements.}

The author would like to thank C.A. Evans and D.A. Reed for valuable discussions and M.I. Danskin and J.K. Hayes for their assistance in preparing this paper.

\section{References}

[1] Castaing R. and Slodzian G., Proc. Eur. Conf. on Electron Microscopy (Delft) Vol. 1 (1960) 169.

[2] SCHUEler B., Proc. SIMS VII Conf. Monterey (Wiley \& Sons 1989) p. 311 and p. 851

[3] SCHUELER B., SANDER P. and REED D.A., Vacuum 41 (1990) 1661.

[4] SCHUeler B., OdOM R.W. and ChaKel J.A., Proc. SIMS VIII Conf. Amsterdam, to be published.

[5] Wollnik H. and MaTSUO T., Int. J. Mass Spectrom. Ion Phys. 37 (1981) 209.

[6] POsCHENRIEDER W.P., Int. J. Mass Spectrom. Ion Phys. 9 (1972) 357.

[7] OETJEN G.H. and PoschenRIEDER W.P., Int. J. Mass Spectrom. Ion Phys. 16 (1975) 353.

[8] SakuraI T., Matsuo T. and MatSUda H., Int. J. Mass Spectrom. Ion Proc. 63 (1985) 273. 
[9] SAKURAI T. et al., Int. J. Mass Spectrom. Ion Proc. 66 (1985) 283.

[10] TAKESHITA I., Z Naturforschg. 21a (1965) 9.

[11] NAKabUSHI H., SAKURAI T. and MaTSUDA H., Int. J. Mass Spectrom. Ion Phys. 52 (1983) 319.

[12] Slodzian G., NBS Spec. Publ. 427 (1975) 33.

[13] Slodzian G., Applied Charged Particle Optics, A. Septier Ed. (Academic Press, New York 1980) p. 1.

[14] Karataev V.I., Mamyrin B.A. and Shmikx D.V., Sov. Phys. - Tech. Phys. 16 (1972) 1177.

[15] Mamyrin B.A., Karataev V.I., ShmikK D.V. and Zagulin V.A., Sov. Phys. -JETP 37 (1973) 45.

[16] GlashChENKo V.P., SEMkIN N.D., SySOEV A.A., Oleinikov V.A. and TATUR V. Yu., Sov. Phys. Tech. Phys. 30 (1985) 540.

[17] Slodzian G. and Figueras A., J. Phys. Lett. France 39 (1978) 90.

[18] SChWieters J., Cramer H.-G., Heller T., JUERgens U., NiEhuis E., ZehNpfenNig J. and BENNINGHOVEn A., J. Vac. Sci. Technol. A9 (1991) 2864.

[19] COATES P.B., Rev. Sci. Instrum. 63 (1992) 2084.

[20] Karas M., Bachmann D., BaHR U. and HillenkaMP F., Int. J. Mass Spectrom. Ion Proc. 78 (1987) 53.

[21] MCFARLANE R.D., HILl J.C., JaCOBS D.L. and PhelpS R.G., Mass Spectrometry in the Analysis of Large Molecules, C.J. McNeal Ed. (Wiley \& Sons Ltd., 1986).

[22] VAN DER PEYL G.J.Q., VAN DER ZANDE W.J., HOOGER BRUGGE R. and KISTEMAKER P.G., Int. J. Mass Spectrom. Ion Proc. 67 (1985) 147.

[23] Hagenhoff B., VAN LEYEN D., Niehuis E. and Benninghoven A., Proc. of the Sixth Int. Conf. on Secondary Ion Mass Spectrom. (SIMS VI) (J. Wiley and Sons 1987) p. 235. 